\title{
Estudio del perfil antropométrico a deportistas de fútbol sala para determinar la posición de juego según el somato tipo e índice de masa corporal
}

\section{Anthropometric profile study of futsal athletes to determine the position of play according to somato type and body mass index}

\author{
MSc, Marco Antonio Toala Pilay ${ }^{1}$ \\ mtoala4561@utm.edu.ec \\ MSc, Elva Katherine Aguilar Morocho ${ }^{2}$ \\ eaguilar@utm.edu.ec
}

Recibido: 1/04/2018, Aceptado: 1/06/2018

\begin{abstract}
RESUMEN
Esta investigación se realizó en la Universidad Estatal del Sur de Manabí (UNESUM), consistió en determinar el perfil antropométrico de deportistas de la disciplina de futbol sala de la selección de la mencionada Institución de Educación Superior, mediante el registro de 16 medidas que corresponden al perfil restringido se determinó el somatotipo y el índice de masa corporal como aspectos elementales para fijar la posición del deportista en el campo de juego, la población de estudio fueron 20 deportistas promedio de 21,20 años, de los cuales fueron 3 porteros, 5 cierres, 6 pívots y 6 alas, se utilizó el método del somatotipo de Heath-Carter, una vez obtenido los valores de los componentes del somatotipo, la representación gráfica se hizo mediante somatocarta calculando las coordenadas $X$ e $Y$, para el análisis en la determinación de la posición en el campo de juego se comparó los valores obtenidos con datos de referencia internacional publicado en estudios similares de esta disciplina deportiva, Los resultados determinaron valores relevantes $(p<0,05)$ en el índice masa corporal entre porteros y aleros, porteros y cierres, y porteros y pívots, sin embargo en la estatura entre ellos no difiere valores relevantes $(P>0,01)$, así mismo se determinó un somatotipo mesomórfico balanceado, en la composición corporal evidenciándose diferencias relevantes $(p<0,05)$ en el índice de masa corporal entre porteros y cierres, para determinar el perfil antropométrico no hay valores relevantes que sean muy significativos en las características antropométricas entre jugadores de la posición de porteros con cierres, pívots y alas.
\end{abstract}

Palabras clave: perfil antropométrico, somatotipo, masa corporal, futbol sala

\footnotetext{
${ }^{1}$ Docente de la Universidad Técnica de Manabí, Ecuador
}

2 Docente de la Universidad Técnica de Manabí, Ecuador 


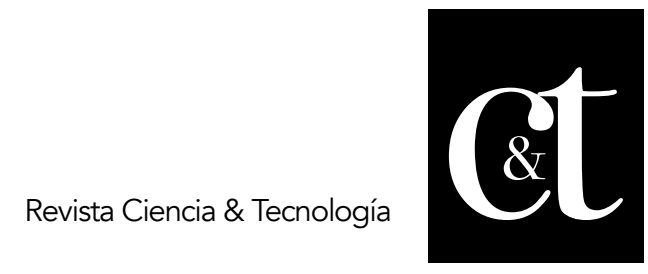

No. 19, 31 de julio de 2018

ISSN impreso: 1390 - 6321

\begin{abstract}
This research was carried out at the Southern State University of Manabí (UNESUM), it consisted of a study to determine the anthropometric profile of soccer athletes of the selection of the mentioned Institution of Higher Education, through the record of 16 measurements that correspond to the restricted profile, the somatotype and the body mass index were determined as elementary aspects to determine the position of the athlete in the field of play, for the study the population was 20 athletes with an average of 21, 20 years, of which 3 were goalkeepers, 5 closures, 6 pivots and 6 wings, the Heath-Carter somatotype method was used, once the values of the somatotype components were obtained, the graphic representation was made with somatocarta calculating the $X$ and $Y$ coordinates, for the analysis in the determination of the position in the field of play the values obtained were compared with data from International reference published in similar studies of this sports discipline, The results determined relevant values $(p<0.05)$ in body mass index between jumpers and goalkeepers, goalkeepers and closers, and goalkeepers and pivots, however in the height between them no Differing relevant values ( $P>0.01)$, likewise a balanced mesomorphic somatotype was determined, body composition revealed relevant differences $(p<0.05)$ in body mass index between goalkeepers and closures, according to this study. To determine the anthropometric profile there are no relevant values that are very significant in the anthropometric characteristics between players in the position of goalkeepers with closures, pivots and wings.
\end{abstract}

Keywords: anthropometric profile, somatotype, body mass, indoor football

\title{
Introducción
}

A nivel del mundo y de manera particular en Sudamérica se han realizado estudios relacionados a perfiles antropométricos en jugadores de futbol sala, sobre todo para determinar el somatotipo y el índice de masa corporal como factor de incidencia en las posiciones en el campo de juego, en este contexto este trabajo es la primera investigación en el futbol sala del Ecuador.

El Ecuador al tener en plena competencia una liga nacional de futbol sala profesional en las series $A$ y $B$, esta investigación es un impulso para realizar posteriormente estudios similares a estos equipos profesionales de futbol sala. Este estudio permite abrir un campo laboral para los profesionales de educación física, ya que los resultados generan un impacto de carácter científico en relación a la importancia del estudio del somatotipo y el índice de masa corporal en los deportistas de manera específica en la disciplina de futbol sala.

En el Cantón Jipijapa no existe antecedentes de estudios de este tipo por lo cual se considera un aporte al desarrollo del deporte cantonal ya que en la actualidad resulta necesario que se establezca en los deportistas de la disciplina de futbol sala de la Universidad Estatal del Sur de Manabí la identificación del somatotipo y composición corporal como un aspecto fundamental en las posiciones del campo de juego ya que de esta forma se facilita el proceso de entrenamiento deportivo de manera óptima.

Para la FIFA (2013) El fútbol sala o futsal como se lo conoce es un deporte colectivo de pelota practicado entre dos equipos de 5 jugadores cada uno, dentro de una cancha de suelo duro, surgió inspirado en otros deportes como el fútbol, que es la 


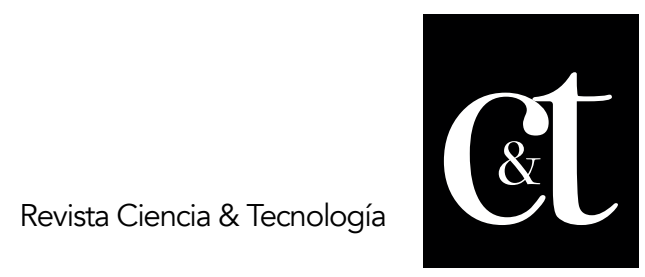

No. 19, 31 de julio de 2018

ISSN impreso: 1390 - 6321

base del juego; el balonmano; y el baloncesto; tomando de estos no solo parte de las reglas, sino también algunas tácticas de juego.

Según lo señala (Otero y Silva, 2015), el fútbol sala aparece como una de las modalidades deportivas más practicadas por la población y sus posiciones en el campo de juego se consideran como porteros, cierres, aleros y pívots. "El fútbol sala, considerado junto a futbol como el deporte más famoso a nivel mundial, consiste en un juego grupal complejo, en el cual, las demandas tanto físicas, antropométricas, así como fisiológicas de los jugadores no son estándar éstas varían de acuerdo a las actividades que se desempeñen dentro del equipo, así como según el nivel de competencia y factores ambientales" (Vera et al., 2014).

"El fútbol sala es un deporte colectivo, entendemos por deporte colectivo aquel juego de conjunto cuyas normas se concretan por medio de un reglamento oficial, que agrupa a varios sujetos con unas características determinadas y los enfrenta a otro grupo con las mismas bases para la obtención de un resultado" (Mora, Zarco y Blanca, 2001).

Para Coyle (2009) los jugadores de fútbol sala tocan el balón con mucha mayor frecuencia hasta seis veces más, con lo que aprenden más deprisa, así mismo el balón al ser más pequeño y pesado, exige un control más preciso, los pases son bien medidos, y el jugador busca ángulos y espacios para realizar combinaciones rápidas con sus compañeros los jugadores que se han formado en el futbol sala poseen un mejor control del balón y una excelente visión de juego (Leonardo, Sospedra, Sanchiz, Mañez y Soriano, 2012).

\section{Desarrollo}

Según Bortoli y De Bortoli (2001), "Cuando dos equipos disputan un partido de fútbol sala, al igual que ocurre con cualquier otro deporte de colaboraciónoposición, debe pretenderse que el resultado sea justo en función de la calidad y cantidad del juego desarrollado en función de la posición de los jugadores en el campo de juego"

Para Castillo-Rodríguez, Madinabeitia, Castillo y Cárdenas (2018) "el futsal, al igual que otros deportes de equipo, requiere una constante toma de decisiones que supone el procesamiento de una gran cantidad de información en un escaso intervalo de tiempo, lo que genera una alta presión cognitiva y emocional."

En el fútbol sala competitivo, el nivel de conocimiento de los entrenadores es bien semejante, en lo que concierne a los aspectos técnicos, tácticos y físicos la diferencia se concreta en los aspectos antropométricos (Franco y Teixeira, 2013). "La preparación condicional de las categorías de formación del fútbol sala carece de una información específica que determine las exigencias que implica la competición" (Prieto, 2006a).

"El estudio de las características morfológicas del perfil antropométrico de los deportistas ha aumentado de forma considerable en los últimos años, realizando aportes significativos para el entrenamiento deportivo, la selección deportiva, la didáctica y pedagogía de la educación física y del deporte" (Jiménez y Molina, 2012). 


\begin{abstract}
"La búsqueda del morfotipo ideal y perfil antropométrico para cada especialidad deportiva es una tarea más compleja en los atletas que participan en deportes de equipo, que en los que participan en deportes individuales" (Benítez y Rojano, 2014) por tanto la diferencia del biotipo del deportista de Europa y otras regiones de América con el deportista ecuatoriano en la disciplina de futbol sala es muy grande, ya que incluso en el Ecuador en un país mega diverso que tiene distintas razas, etnias y regiones por lo cual resulto necesario determinar el somatotipo ideal del deportista de futbol sala de UNESUM, la cual es una universidad que acoge a estudiantes de diversas poblaciones del Ecuador.
\end{abstract}

"El estudio del perfil antropométrico de poblaciones determinadas posibilita la obtención de forma fácil de datos que tienen importancia tanto para la obtención de tipologías como para la prescripción de entrenamientos" (Gil y Verdoy, 2011). "Las características para determinar el perfil antropométrico son parte del conjunto de variables biológicas relacionadas con el rendimiento deportivo" (Rienzi y Mazza, 1998) este perfil está determinado por la cineantropometría.

La Cineantropometría es un área básica dentro de la valoración médica del deportista (Fernández y Alvero, 2006) "La cineantropometría es una disciplina científica que ha sido utilizada en áreas como la nutrición, la antropología, la ergonomía, la biomecánica, la endocrinología y la medicina" (Albureque, 2005). Existen bastantes estudios en los que se han obtenido diferentes perfiles antropométricos en función de la posición que ocupan los jugadores (Berral de la Rosa, 1999).

"Cada una de las modalidades deportivas existentes poseen unas características propias que la hacen diferente del resto de especialidades deportivas, por lo que los atletas de cada modalidad deportiva tendrán un patrón cineantropometrico específico que se adaptará mejor a las características propias del deporte que practiquen" (Prieto, 2006b). "La cineantropometría es el estudio del tamaño, la forma, la proporcionalidad, la composición, la maduración biológica y la función corporal; con objeto de entender el proceso del crecimiento, el ejercicio, el rendimiento deportivo y la nutrición" (Ross \& Marfell-Jones, 1991).

"El somatotipo hace referencia de una forma fotográfica al perfil del deportista respecto a tres componentes, la endomorfia, la mesomorfia y la ectomorfia" (Martínez, Undanpilleta, Guerrero y Barrios, 2011).

"La representación gráfica del somatotipo se realiza en la somatocarta, donde se pueden comparar diferentes mediciones del mismo deportista, o diferentes grupos y ver su evolución" (Cejuela, 2019).

Según el método de Heath-Carter. "el somatotipo es una descripción numérica de la configuración morfológica de un individuo en el momento de ser estudiado donde influye factores exógenos como: edad, sexo, crecimiento, actividad física, alimentación, factores ambientales, medio socio-cultural, raza" (Carter, 1996).

Según lo afirman Cosio-Bolaños et al. (2011), "La estimación del índice de masa corporal es de interés en diversas áreas como la nutrición, la medicina, la 
antropología y las ciencias del deporte."

\section{Materiales y métodos}

Participantes Se evaluaron 20 deportistas con un promedio de edad de 21,20 años, mismos que conforman la selección de futbol sala de la Universidad Estatal del Sur de Manabí, a todo el grupo se le informo del objetivo de la presente investigación, por lo cual firmaron una carta de aceptación.

Las medidas que corresponden al perfil restringido fueron tomadas por los autores para lo cual el primer autor asistió y aprobó el curso de International Society for the Advancement Kineanthropometry (ISAAK) nivel 1 y las 20 medidas corresponden a normas de aprobación por lo cual las 20 medidas de las muestras fueron validadas por el Mg. Eduardo Brito P, antropometrista nivel 3 de ISAAK.

\section{Materiales}

Los materiales que se utilizaron fueron los siguientes:

a) Tallímetro (precisión: 1mm)- bascula (precisión: $100 \mathrm{gr}$ ) marca seca.

b) Cinta antropométrica metálica inextensible (precisión: $1 \mathrm{~mm}$ ) marca calibres argentinos.

c) Paquímetro de diámetros óseos pequeños (precisión: 1mm) marca calibres argentinos.

d) Paquímetro de diámetros óseos grandes (precisión: 1mm) marca calibres argentinos.

e) Plicómetro (precisión: 1,2mm) marca calibres argentinos.

f) Lápiz demográfico para marcar en el lado derecho los puntos anatómicos.

Se tomaron las siguientes medidas:

- Peso $(\mathrm{kg})$ - talla $(\mathrm{cm})$

- Tríceps - Subescapular - Bíceps - Cresta Iliaca - Supraespinal - Abdominal Muslo medial - Pierna (pantorrilla)

$\square$ perímetros (cm) Brazo relajado - Brazo flexionado y contraído - Cintura (min.) - Cadera (max.) - Pierna (max.)

$\square$ diámetros $(\mathrm{cm}) \quad$ Húmero (biepicondíleo) - Fémur (bicondíleo)

- Pliegues $(\mathrm{mm})$

\section{Procedimiento}

La valoración se realizó en dos días continuos 18 y 19/09/2018 en la jornada de la mañana en el rango de 4 horas desde las 8 h30 hasta las $11 \mathrm{~h} 30$ sin haber realizado ninguna práctica de actividad física durante las 24 horas anteriores a la valoración, en la medición se consideró el error técnico de medición (ETM) intra - observador según ISAAK para el nivel 1 (7,5\% para pliegues y $1,5 \%$ para perímetros, diámetros, altura y peso.

El peso corporal ideal se lo obtuvo a partir del índice de masa corporal (IMC $=$ peso $(\mathrm{kg}) /$ talla $(\mathrm{m}) 2$ ) (Márquez y Garatachea, 2002). El estudio del somatotipo se lo valoró siguiendo el método antropométrico HeathCarter identificando los tres componentes como son: Endomorfia, Mesomorfia y Ectomorfia siguiendo la representación gráfica de la somatocarta (X, Y) según Carter (1996).

Acero (2002), a partir de las fórmulas matemáticas, obtiene los tres componentes de somatotipo: En la endomorfia la fórmula es la siguiente: $\mathrm{I}=\left(0,1451^{*} \mathrm{Ss}\right)$ - 
$(0,00068 *$ Ss2 $)+(0,0000014 *$ Ss3 $)-0,7182$ Ss $=$ (tríceps + Subescapular + Suprailiaco) $* 170,8) /$ Talla

Para obtener la mesomorfia se aplicó la siguiente formula: $\mathrm{Me}=(0,858 * \mathrm{DH})+$ $(0,601 * \mathrm{DF})+((0,188 *(\mathrm{BE}-(\mathrm{Tr} / 10)))+((0,161 *(\mathrm{PM}-(\mathrm{MD} / 10)))-(0,131 * \mathrm{Ta})+$ 4,5 Donde se aplica la siguiente simbología $\mathrm{DF}=$ diámetro del fémur $\mathrm{BE}=$ Perímetro bíceps contraído $\mathrm{Tr}=$ Pliegue del tríceps $\mathrm{PM}=$ Perímetro de pierna $\mathrm{MD}=$ Pliegue medial pierna $\mathrm{Ta}=$ Talla

Para obtener la ectomorfia se utilizó la siguiente formula: $E C=(0,732 *$ RPI $)-$ 28,58 para RPI $>40,75 \mathrm{EC}=(0,463 * \mathrm{RPI})-17,63$ para RPI $<38,25$ y RPI $>$ 40,74 . Donde se aplica la siguiente simbología: RPI = Talla / Raíz cúbica del Peso Según en la región que se establezca el punto de coordenadas $X$ e $Y$, este tendrá un significado (Cabañas, Mestre y Herrero, 2009) (ISAAK, 2011). A. Mesomorfo balanceado: La mesomorfia es la dominante, mientras que la endomorfia y la ectomorfia son iguales, sin diferenciarse en más de 0,5. B. Endomorfo balanceado: La endomorfia es dominante, mientras que la mesomorfia y ectomorfia son iguales, sin diferenciarse en más de 0,5. C. Ectomorfo balanceado: La ectomorfia en la dominante, mientras que la mesomorfia y la endomorfia son iguales, sin diferenciarse en más de 0,5. D. Mesomorfo-Endomorfo: La endomorfia y la mesomorfia son iguales, o no se diferencian en más de 0,5, y la ectomorfia es menor. E. Mesomorfo-Ectomorfo: La ectomorfia y la mesomorfia son iguales, o no se diferencian en más de 0,5, y la endomorfia es menor.

F. Endomorfo-Ectomorfo: La endomorfia y la ectomorfia son iguales, o no se diferencian en más de 0,5, y la mesomorfia es menor.

Las posiciones de la $G$ a la $L$, se nombran con el prefijo del componente más alejado y, como sufijo, el nombre del componente más cercano: A. MesoEndomorfo: La endomorfia es dominante y la mesomorfia es mayor que la ectomorfia. B. Endo- Mesomorfo: La mesomorfia es dominante y la endomorfia es mayor que la ectomorfia.

C. Ecto-Mesomorfo: La mesomorfia es dominante y la ectomorfia es mayor que la endomorfia. D. Meso-Ectomorfo: La ectomorfia es dominante y la mesomorfia es mayor que la endomorfia. E. Endo-Ectomorfo: La endomorfia es dominante y la endomorfia es mayor que la mesomorfia. F. Ecto-Endomorfo: La endomorfia es dominante y la ectomorfia es mayor que la mesomorfia.

Análisis estadístico de datos Para el análisis de los datos estadísticos el procesamiento de la información se realizó bajo el sistema operativo Windows 7 Home Basic, con un procesador Intel core i3, para tal efecto se diseñó una hoja de cálculo en el programa Microsoft Excel versión 2010, en la que se organizaron los datos por posición de juego (porteros, cierres, pívots y alas). Para el análisis de las variables se utilizó el programa "Statistical Package for Social Sciences" (SPSS) 19.0 para Windows, para el análisis de verificación de la distribución normal de los datos se aplicó la prueba de Kolmogórov-Smirnov, así mismo se calculó las medidas de tendencia central (promedio, desviación estándar y coeficiente de variación y rango) medidas de posición (percentil) y el análisis de la varianza de un solo factor (ANOVA). 


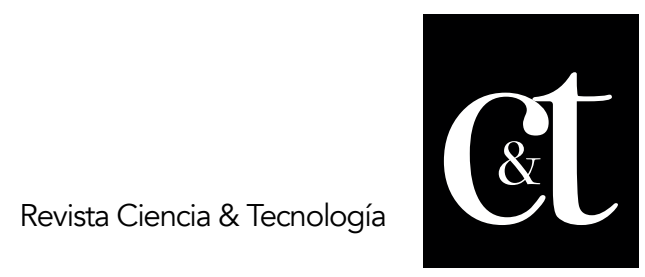

No. 19, 31 de julio de 2018

ISSN impreso: 1390 - 6321

\begin{abstract}
Resultados
En relación al somatotipo en los porteros como se evidencia en el gráfico 1 los deportistas de la selección de UNESUM el promedio es sus características son Mesomorfo balanceado, teniendo una no muy significativa dispersión en los caracteres mesomórficos, y que en relación al promedio mundial el gráfico 5 de porteros guarda una importante relación en al menos un deportistas, así mismo según los gráficos $1,2,3$ y 4 se evidencia una relación antropométrica muy significativa $(p>01)$ entre porteros, ala y pívots, más sin embargo si se distancia la relación $(p<05)$ entre porteros y cierres según los gráficos 1 y 2 en relación al índice de masa corporal según el gráfico 9 el promedio es de 22,24 por lo cual están dentro del rango del peso normal considerado ideal.
\end{abstract}

En relación a la estatura el promedio de los porteros de la selección de futsal de UNESUM es de $168,3 \mathrm{~cm}$ con lo cual la relación $(p<05)$ difiere considerablemente del promedio mundial de jugadores en esta posición de juego, sin embargo, existe una estrecha relación ( $p>01$ ) entre jugadores porteros, pívots y alas.

En relación al somatotipo de los deportistas de la posición denominada cierres sus características son Mesomorfo balanceado según gráfico 2 aunque entre ellos sesga la tendencia a la mesomorfia-endomorfia, sin embargo hay una diferencia significa $(p<05)$ entre las demás posiciones de juego, así mismo en relación al promedio mundial grafico 6 de defensas y cierres guarda una importante relación en al menos dos deportistas, y en relación al índice de masa corporal según gráfico 9 el promedio es de 21,68 por lo cual están dentro del rango del peso normal considerado ideal.

En relación a la estatura el promedio de los jugadores cierres de la selección de futsal de UNESUM es de $176,2 \mathrm{~cm}$ con lo cual la relación $(p>01)$ no difiere del promedio mundial de jugadores en esta posición de juego.

En relación al somatotipo de los deportistas de la posición denominada pívots sus características son Mesomorfo balanceado según el gráfico 3 mostrando una significativa dispersión en este rango, en este aspecto existe una gran relación $(p>01)$ en sus características morfológicas, así mismo en relación al promedio mundial el gráfico 7 de pívots guarda una importante relación en al menos 1 deportista, y en relación al índice de masa corporal según el gráfico 9 el promedio es de 23,48 por lo cual están dentro del rango del peso normal considerado ideal.

En relación a la estatura el promedio de los jugadores pívots de la selección de futsal de UNESUM es de $164,1 \mathrm{~cm}$ con lo cual la relación $(p<05)$ difiere considerablemente del promedio mundial de jugadores en esta posición de juego. En relación al somatotipo de los deportistas de la posición denominada alas sus características son Mesomorfo balanceado aunque se observa según gráfico 4 el somatotipo medio endo-mesomórfico por lo cual se muestra una significativa dispersión en este rango con un deportistas, en este aspecto existe una gran relación $(p>01)$ en sus características morfológicas, así mismo en relación al promedio mundial grafico 8 de alas guarda una importante relación en al menos 2 deportistas, y en relación al índice de masa corporal según el gráfico 9 el promedio es de 21,82 por lo cual están dentro del rango del peso normal considerado ideal.

En relación a la estatura el promedio es de $165,9 \mathrm{~cm}$ con lo cual la relación $(p<05)$ 204 


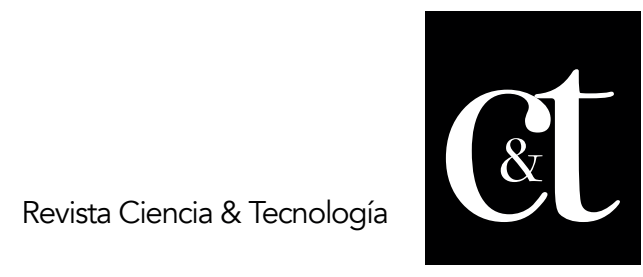

No. 19, 31 de julio de 2018

ISSN impreso: 1390 - 6321

difiere considerablemente del promedio mundial de jugadores en esta posición de juego.

Somatocarta de porteros

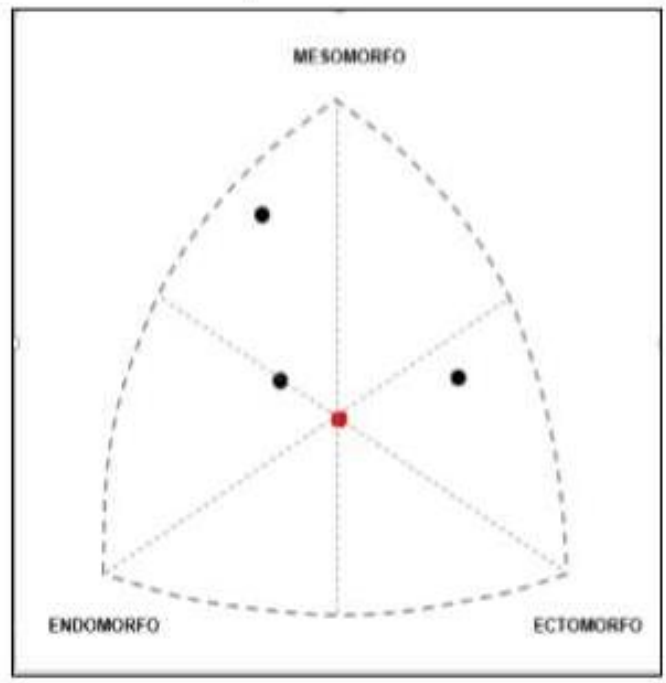

Gráfico 1: FUENTE: EL AUTOR

Somatocarta de pivots

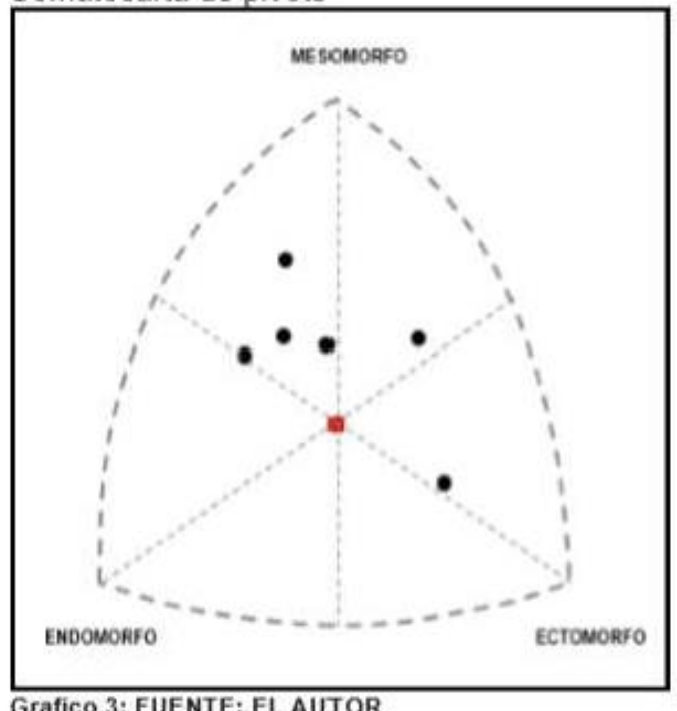

Grafico 3: FUENTE: EL AUTOR
Somatocarta de cierres

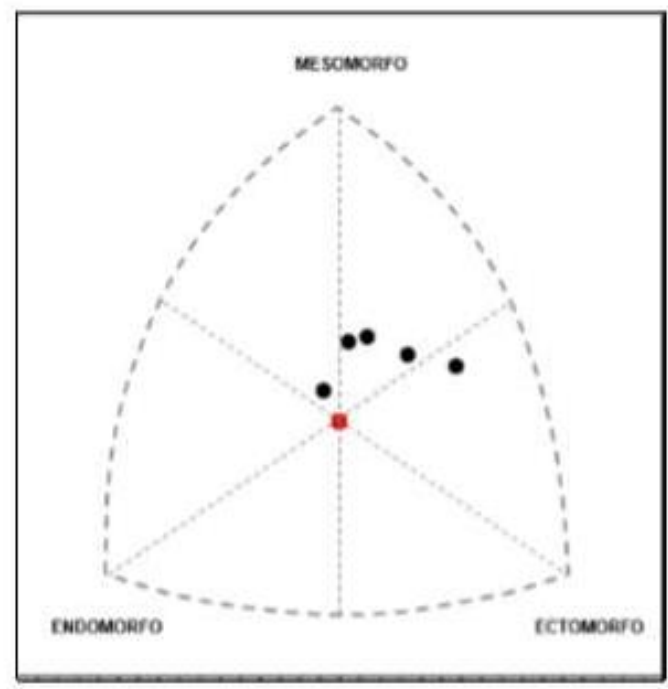

Gráfico 2: FUENTE EL AUTOR

Somatocarta de Alas

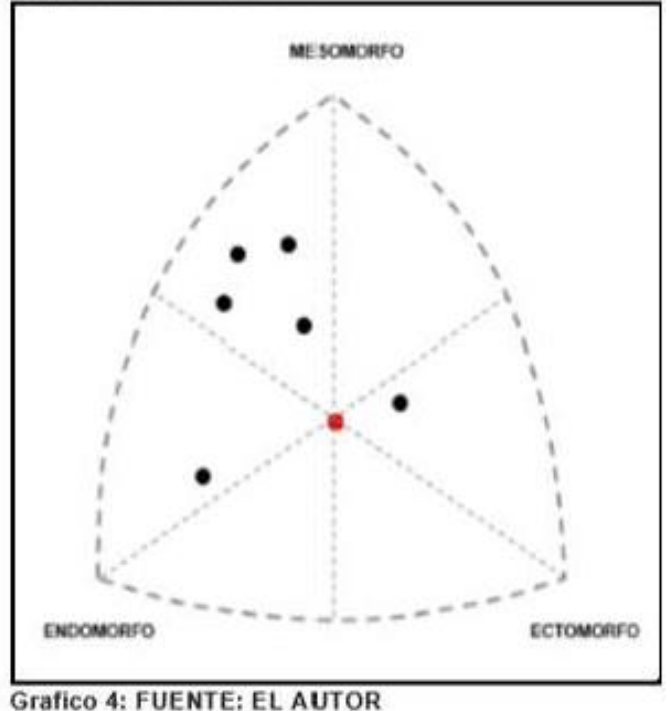

Porcentaje del indice de masa corporal según posición de juego

\begin{tabular}{|l|c|}
\hline POSICION & IMC \\
\hline PORTEROS & 22,24 \\
\hline CIERRES & 21,68 \\
\hline PIVOTS & 23,48 \\
\hline ALAS & 21,82 \\
\hline PROMEDIO & $\mathbf{2 2 , 3 0}$ \\
\hline
\end{tabular}

Grafico 9: FUENTE: EL AUTOR 
ANALISIS COMPARATIVO CON PROMEDIOS MUNDIALES ENTRE PORTEROS

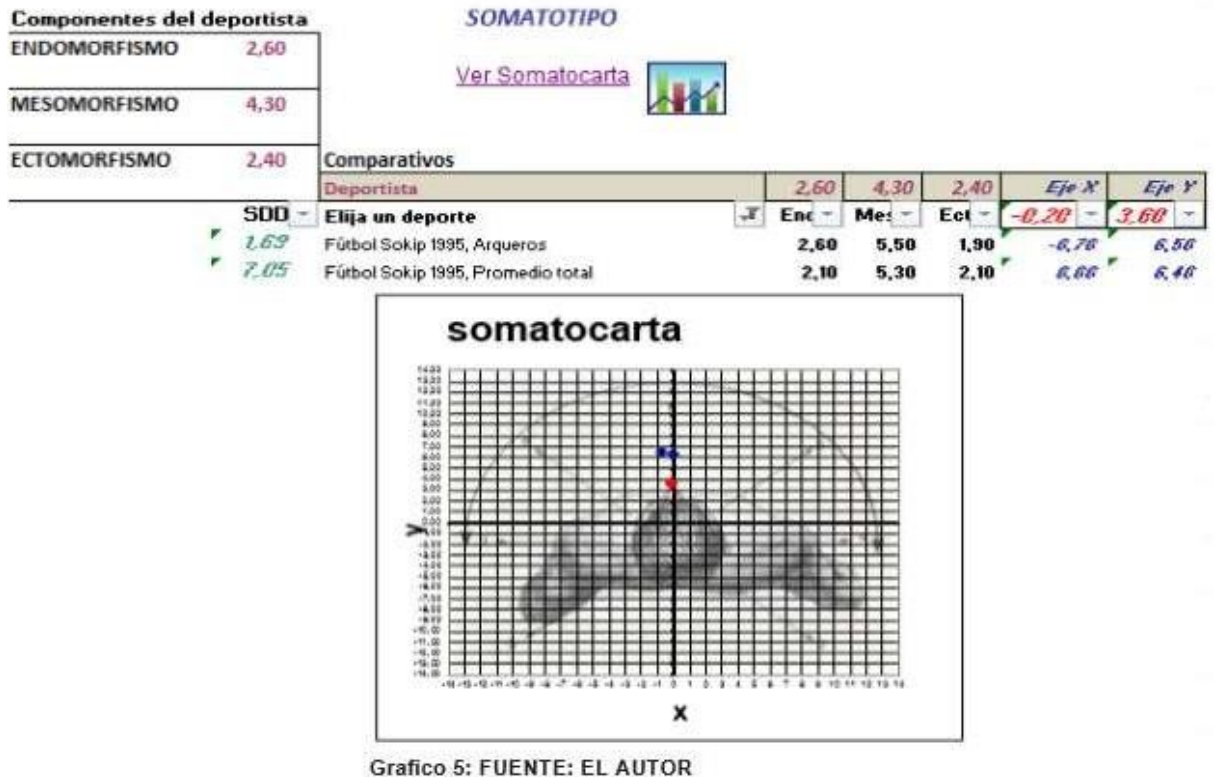

ANALISIS COMPARATIVO CON PROMEDIOS MUNDIALES ENTRE CIERRES enesenuas reamiLs ensele reneron inoicrs acriacnsias

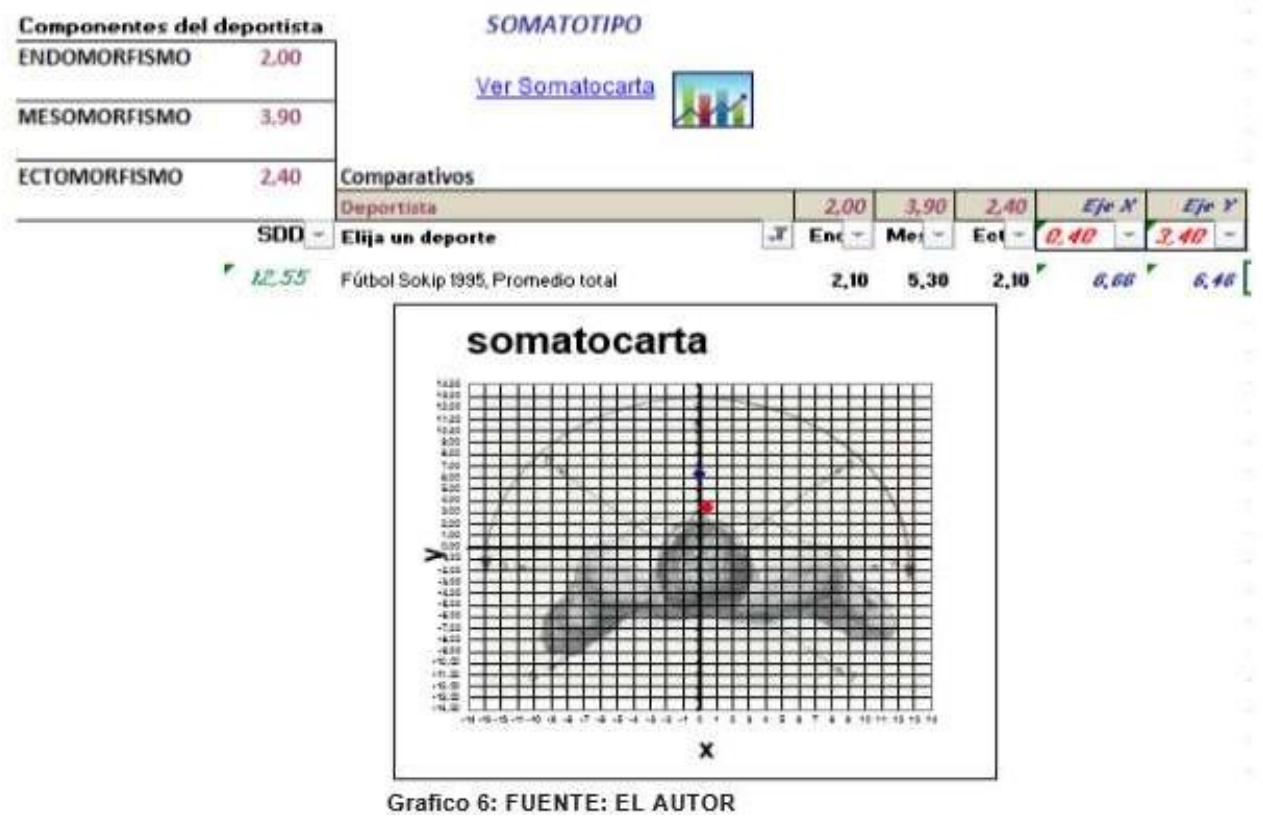




\section{ANALISIS COMPARATIVOS CON PROMEDIOS MUNDIALES ENTRE PIVOTS}

ERESEMIACI RLAMHLA

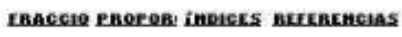

\begin{tabular}{|c|c|c|c|}
\hline \multicolumn{2}{|c|}{ Componentes del deportista } & \multicolumn{2}{|l|}{ SOMATOTIPO } \\
\hline ENDOMORFISMO & 2,70 & \multirow{2}{*}{$\underline{\text { Ver Somatocarta }}$} & \\
\hline MESOMORFISMO & 4,10 & & \\
\hline \multirow[t]{5}{*}{ ECTOMORFISMO } & 1,70 & Comparativos & \\
\hline & & Deportista & \\
\hline & SDD - & Elija un deporte & \\
\hline & $=222$ & Fútbol Sokip 1995, Delanteros centrales & \\
\hline & $\therefore 10,77$ & Fútbol Sokip 1995, Promedio total & \\
\hline
\end{tabular}

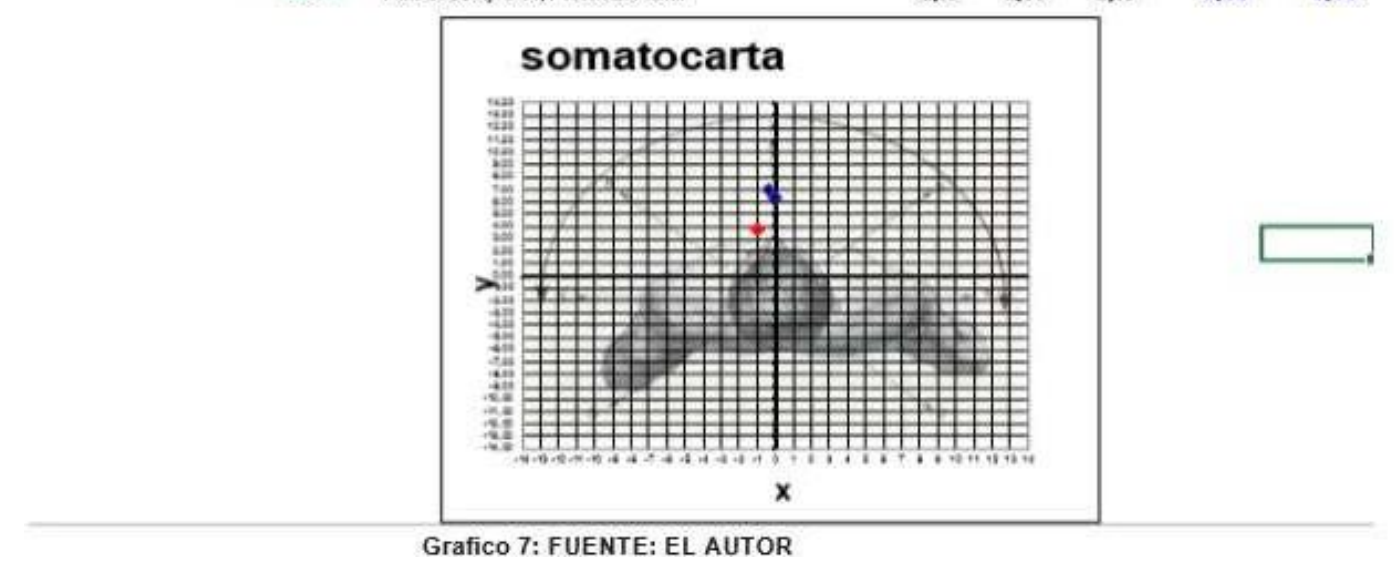

$\mathrm{x}$

Grafico 7: FUENTE: EL AUTOR

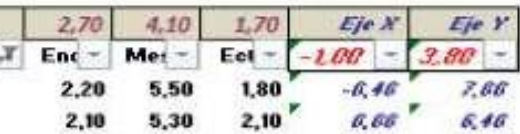

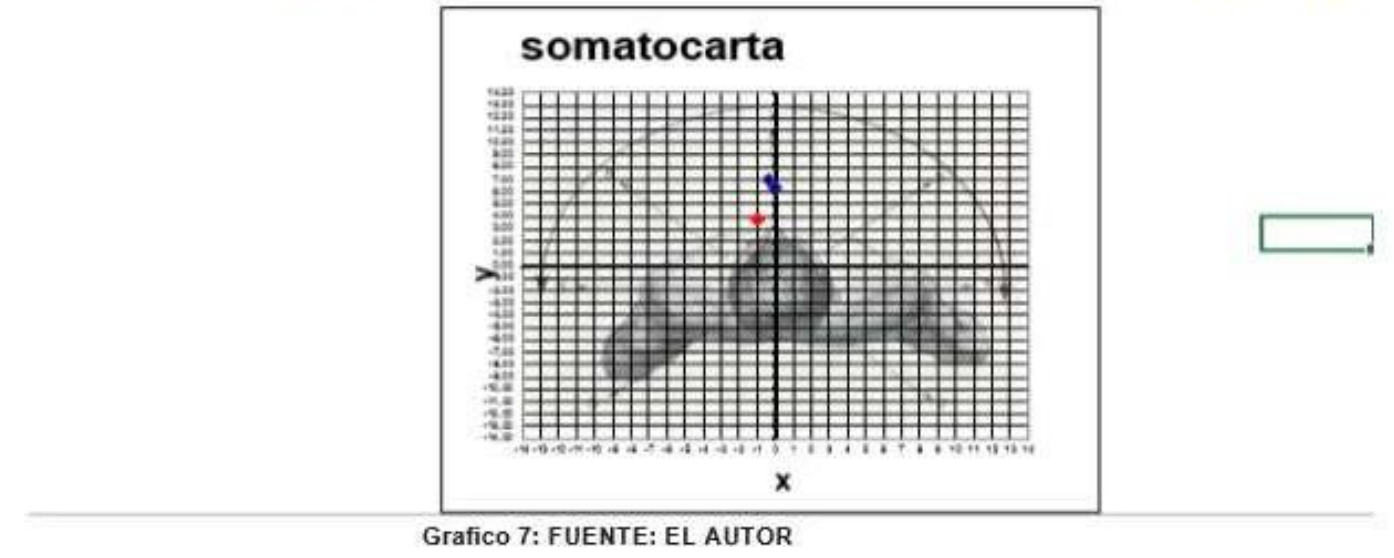

ANALISIS COMPARATIVOS CON PROMEDIOS MUNDIALES ENTRE ALAS
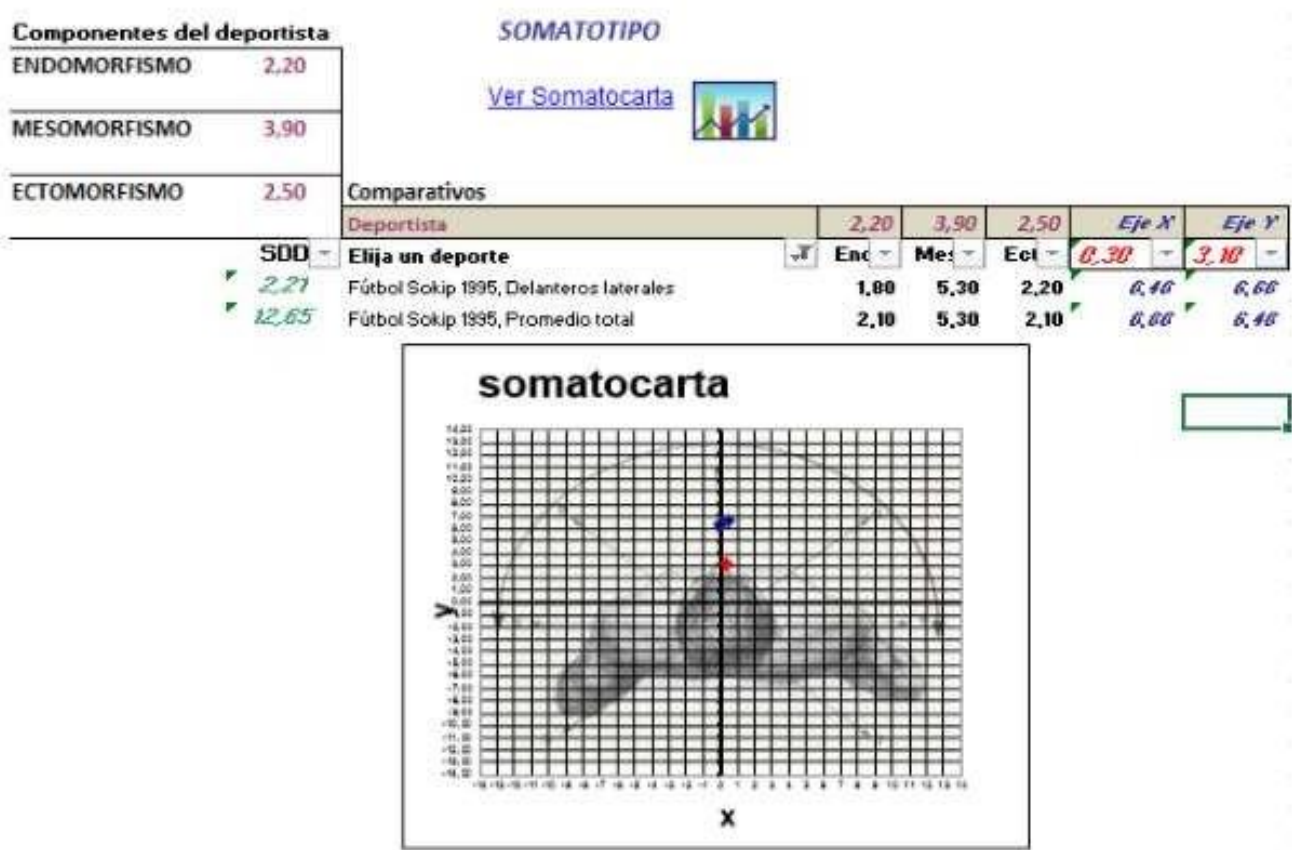

Grafico 8: FUENTE: EL AUTOR

207

Toala, Aguilar. Estudio del perfil antropométrico a deportistas de fútbol sala para determinar la posición de juego según el somato tipo e índice de masa corporal 
La homogeneidad del somatotipo para jugadores según las diferentes posiciones de juego de acuerdo a otros estudios difiere de los resultados en los deportistas de la selección de futbol sala de UNESUM, considerando que como queda evidenciado en los gráficos $1,2,3, y 4$ el somatotipo mesomórfico predominante el rango es muy distante en relación a los promedios con estándares internacionales grafico $5,6,7, y$ 8 , sin embargo la investigación refleja un índice $(p>01)$ que al menos un deportista por posición de juego (porteros, cierres, pívots y alas) se acerca a los promedios estándar internacionales.

En el perfil básico la estatura y el peso, aunque se ubican en el rango mínimo del promedio de todas las posiciones de juego, sin embargo, la investigación presenta un índice $(p<05)$ que difieren considerablemente con los con estándares internacionales grafico 9.

En el perfil de los diámetros, perímetros y pliegues cutáneas, aunque están el rango mínimo $(p<05)$ estos difieren considerablemente por posición de juego con los con estándares internacionales grafico $5,6,7$, y 8 , en los cuales se puede entender que a lo mejor intervienen otros factores como genéticos, alimentación, ambiente, cultura y otros, que tienen alta incidencia para el desarrollo del biotipo.

Al analizar los resultados en cuanto al índice de masa corporal grafico 9 se evidencia una clasificación de normopeso para todas las posiciones de juego en el futbol sala (porteros, cierres, pívots y alas), ya que se encuentran dentro del rango de normalidad planteado por la Organización Mundial de la Salud para personas adultas que va de 18 a $25 \mathrm{~kg} / \mathrm{m} 2$. No obstante en la investigación queda evidenciado que el $10 \%$ de los evaluados se encuentran en riesgo de sobrepeso.

\section{Conclusiones}

Los resultados determinaron valores relevantes $(p<0,05)$ en el índice masa corporal entre porteros y alas, porteros y cierres, y porteros y pívots, sin embargo en la estatura entre ellos no difiere valores relevantes $(P>0,01)$, así mismo se determinó un somatotipo mesomórfico balanceado, según este estudio para determinar el perfil antropométrico no hay valores relevantes que sean muy significativos en las características antropométricas entre jugadores de la posición de porteros con cierres, pívots y alas de la selección de futbol sala de UNESUM, sin embargo existe un sesgo mínimo del promedio estándar internacional por cada posición de juego.

\section{Referencias bibliográficas}

Acero, J. (2002). Cineantropometria: Fundamentos y procesos. Cali: Escuela sin fronteras.

Albureque, F. S. (2005). Kinanthropometric assessment of a football team over one season. European Journal of Anatomy, 17-22.

Benítez, A. y Rojano, D. (2014). Características cineantropométricas de jugadores de fútbol de categoría amateur en relación a la posición de juego. Revista Internacional de Deportes Colectivos, 5-19.

Berral de la Rosa, F. G. (1999). Somatotipo. Revista Uruguaya de medicina del ejercicio, 14-28.

Bortoli, A. y De Bortoli, R. y. (2001). Utilización de coeficientes ofensivos para el análisis del rendimiento deportivo en el futbol sala. European Journal of Human 


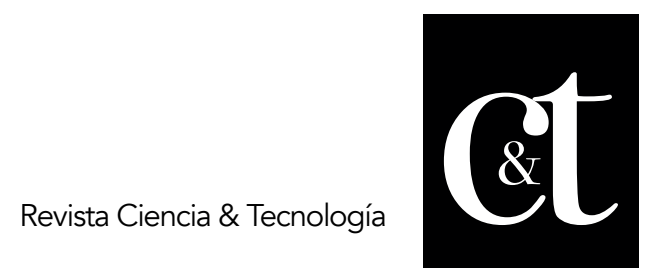

No. 19, 31 de julio de 2018

ISSN impreso: 1390 - 6321

\section{Movement, 7-17.}

Cabañas, H., Mestre, L. y Herrero, L. (2009). Introducción de la técnica antropométrica. Método. Medidas antropométricas. Puntos anatómicos. Madrid: Editores. Compendio de Cineantropometría.

Carter, L. (1996). Somatotipo. Sydney-Australia: Editores Antrhopometrica. University of New South Wales Press.

Castillo-Rodríguez, A., Madinabeitia, I., Castillo, A. y Cárdenas, D. (2018). La impulsividad determina el rol desempeñado por los jugadores de futsal. Revista de Psicología del Deporte/Journal of Sport Psychology, 27(2): 181-188.

Cejuela, R. (2019). Valoración antropométrica: el somatotipo. Sport Training Magazine, 26-31.

Cosio-Bolaños, M., Dearruda, M., Moyano, A., Gañan, E., Pino, L. y Lancho, J. (2011). Composición corporal de jóvenes universitarios en relación a la salud. Nutrición clínica y dietética hospitalaria, 15-21.

Coyle, D. (2009). Las Claves del Talento. Madrid: Zenith Editorial.

Fernández, S., y Alvero, J. (2006). La producción científica en cineantropometría: Datos de referencia de somatotipo y composición corporal. Archivo de Medicina del Deporte, 17-35.

FIFA (del 1 al 30 de octubre de 2013). Página oficial de la Fédération Internationale de Football Association (FIFA). Obtenido de http://fifa.com.

Franco, N. y Teixeira, T. D. (2013). El liderazgo de los entrenadores de fútbol sala. Revista de Psicología del Deporte, 11-17.

Gil, J. y Verdoy, P. (2011). Caracterización de deportistas universitarios de fútbol y baloncesto: antropometría y composición corporal. E-balonmano.com: Revista de Ciencias del Deporte, vol. 7, núm. 1, 2011, pp. 39-51.

Jiménez, C. y Molina, L. (2012). Estudio comparativo de la composición corporal y el somatotipo entre jugadores de fútbol sala universitario y profesional de la región suroccidente de Colombia. Cali: Universidad del Valle.

Leonardo, R., Sospedra, I., Sanchiz, I., Mañez, J. y Soriano, J. (2012). Comparación del somatotipo, evaluación nutricional e ingesta alimentaria entre estudiantes universitarios deportistas y sedentarios. Medicina Clínica, 54-60.

Márquez, S. y Garatachea, N. (2002). Actividad física y salud. Funieber.

Martínez, J., Undanpilleta, A., Guerrero, J. y Barrios, V. (2011). El somatotipomorfología en los deportistas. ¿Cómo se calcula? ¿Cuáles son las referencias internacionales para comparar con nuestros deportistas? efdeportes, 17-25.

Mora, J., Zarco, J. y Blanca, M. (2001). Atención -concentración como entrenamiento para la mejora del rendimiento deportivo en jugadores de futbol profesional. Revista de Psicología del Deporte, 49-65.

Otero, F. y Silva, J. (2015). Fiabilidad en la observación en fútbol sala a través de una herramienta de evaluación de los deportes de equipo. Revista Iberoamericana de psicología del ejercicio y el deporte, vol. 10, núm. 2, 2015: 259-266. 
Prieto, I. (2006a). Composición corporal de jugadores. efdeportes, 1-10.

Prieto, I. (2006b). Las capacidades condicionales en el joven jugador de futbol sala. efdeportes, 1-7.

Rienzi, E. y Mazza, J. (1998). Dimensiones corporales absolutas del futbolista sudamericano. Futbolista sudamericano de élite. Ed. Biosystem servicio educativo. pp., 33-48.

Ross W.D. \& Marfell-Jones M.J. (1991). Kinanthropometry. En: MacDougall JD, Wenger HA, Green HJ, editors. Physiological testing of elite athlete. London: Human Kinetics, 1991. p. 223-308.

Vera, Y., Chávez, C., David, A., Torres, W., Rojas, J. y Bermúdez, V. (2014). Características morfológicas y somatotipo en futbolistas no profesionales, según posición en el terreno de juego. Revista Latinoamericana de Hipertensión, 1320. 